\title{
Cáncer gástrico, epidemiología y prevención
}

\author{
Rafaela Sierra'
}

\begin{abstract}
Resumen: El cáncer gástrico es una de las neoplasias que produce mayor mortal idad en la población mundial . A pesar de que las tasas de incidencia están disminuyendo, sigue siendo un problema de salud pública. La información y el conocimiento sobre la epidemiología de este tumor es abundante. A quí se presenta una revisión bibliográfica actualizada sobre los factores de riesgo mejor documentados y aceptados por la comunidad científica: infección por Helicobacter pylori, dieta, susceptibilidad genética y nivel socioeconómico. A demás, se analizan formas de enfrentar esta patología, por medio de la prevención (consumo de frutas y verduras) y la detección temprana con Rayos $X$ y marcadores biológicos (pepsinógenos séricos), en poblaciones o grupos identificados como de alto riesgo. Se hace hincapié en las interrogantes y retos que plantean los nuevos conocimientos.
\end{abstract}

Descriptores: Cáncer gástrico, Helicobacter pylori, susceptibilidad genética, interleucinas, dieta, prevención, detección temprana, pepsinógenos.

Recibido: 15 de enero, 2002

Aceptado para publicación: 23 de abril, 2002

A pesar del descenso continuo de las tasas de incidencia y mortalidad, por cáncer gástrico, este continúa siendo la segunda causa de muerte por cáncer en el mundo․ En el año 2000 el número de casos nuevos fue mayor de un millón, dos tercios de los cuales aparecieron en los países en desarrollo; ese número aumenta cada año debido al envejecimiento de la población. L as tasas más altas se presentan en Japón, China, Corea, países del Este de Europa, países tropicales deA mérica del Sur y Costa Rica. Las tasas más bajas ocurren en la población blanca de los Estados Unidos, A ustralia y Á frica ${ }^{1-3}$.

La supervivencia de los pacientes con cáncer gástrico es muy baja, tanto en los países industrializados como en los que están en vías de desarrollo, debido a que la mayoría de los casos se diagnostican en etapas avanzadas, cuando la probabilidad de curación es muy reducida ${ }^{4,5}$. En Japón, las tasas generales de supervivencia son considerablemente mayores gracias a los programas masivos de detección temprana, lo que permite aplicar el tratamiento oportuno $0^{6,7}$.

\footnotetext{
A breviaturas: $P$ G $=$ Pepsinógeno; $V$ acA $=$ Citotoxina vacuolizante $A$; cagA $=$ gen $A$ asociado a citotoxina.

Instituto de Investigaciones en Salud (INISA). Universidad de Costa Rica
}

Correspondencia: Rafaela Sierra. Instituto de Investigaciones en Salud (INISA ). U niversidad de Costa Rica. e-mail: rsierra@ cariari.ucr.ac.cr Fax: (506) 207-51-30
La mayoría de los tumores del estómago son de origen epitelial $(97 \%)$, se clasifican como adenocarcinomas y han sido divididos en dos principales tipos: difuso e intestinal. Difieren en su epidemiología, etiología, patogénesis y comportamiento. El adenocarcinoma de tipo difuso o indiferenciado, produce metástasis más temprano, se presenta por igual en toda la población, es más frecuente en poblaciones jóvenes que el intestinal y está más relacionado con factores genéticos ${ }^{8-10}$.

El adenocarcinoma de tipo intestinal es bien diferenciado, de crecimiento expansivo y es prevalente en poblaciones de alto riesgo de cáncer gástrico y en hombres de mayor edad. Se ha relacionado con factores ambientales. La disminución de la incidencia de cáncer gástrico se atribuye principal mente a la disminución del tipo intestinal. Los cambios que preceden a la aparición de este tipo de cáncer son la gastritis crónica atrófica, la metaplasia y la displasia. El proceso completo tarda por lo menos dos décadas ${ }^{11,12}$. En todas las etapas de este proceso interactúan la predisposición genética, los factores de riesgo y los factores de protección, facilitando, retrasando o evitando el desarrollo del cáncer .

\section{Factores de riesgo y protección}

La infección con Helicobacter pylori, especialmente desde edades tempranas, Ia historia familiar de cáncer gástrico, el bajo nivel socioeconómico, la producción endógena de nitrosaminas, la alta ingesta de compuestos nitrosados e irritantes y el bajo consumo de frutas y verduras, han sido asociados con mayor riesgo de padecer cáncer gástrico de 
tipo intestinal. Las variaciones geográficas en las tasas de incidencia de este tumor, las tendencias temporales decrecientes y las variaciones del riesgo en las poblaciones que emigran, sugieren que existen factores ambientales que cumplen un papel importante en la carcinogénesis gástrica.

\section{H eliocobacter pylori y cáncer gástrico}

Se reconoce que la infección con Helicobacter pylori es la principal causa de gastritis crónica, úlceras pépticas y el principal factor de riesgo para el desarrollo de cáncer gástrico. La infección se adquiere, en la mayoría de los casos, en la infancia, y es capaz de permanecer en el hospedero toda la vida. Sin embargo, solo un grupo muy pequeño desarrolla cáncer gástrico o úlceras pépticas, mientras que un gran número de infectados (más del $70 \%$ ) son asintomáticos ${ }^{13-17}$. Los aspectos más relevantes de la infección son: la inflamación crónica, la proliferación celular excesiva y la disminución de la secreción ácida, lo cual puede modular el proceso de carcinogénesis $\mathrm{s}^{18-20}$. Surge la pregunta sobre cuáles son las circunstancias o mecanismos que predisponen al hospedero infectado para que desarrolle las patologías gástricas más o menos severas.

A Igunos estudios sugieren la existencia de cepas de $\mathrm{H}$. pylori más virulentas, de acuerdo con algunos fenotipos de la misma. Las cepas productoras de la toxina VacA y las que poseen el gen cagA, han sido asociadas con mayor riesgo de metaplasia intestinal y cáncer gástrico ${ }^{21,22}$. Sin embargo, algunas investigaciones encontraron que la fuerza de la asociación varía entre diferentes poblaciones, o no se encuentra asociación ${ }^{23,24}$, ni se han podido identificar en la bacteria, los genes o polimorfismos de mayor riesgo para úlceras 0 cáncer ${ }^{25,26}$. A ctualmente, varios factores de virulencia de la bacteria, están siendo ampliamente investigados.

Por otra parte, investigaciones recientes muestran que los pacientes infectados y que presentan gastritis atrófica severa, gastritis predominante en el cuerpo o metaplasia intestinal, tienen mayor riesgo de cáncer gástrico, así como también aquellas personas con úlceras gástricas 0 pólipos hiperplásicos. Pero no existe ese riesgo entre los pacientes con úlceras duodenales ${ }^{27}$. Se ha postulado que las enfermedades gástricas, asociadas con la infección por H. pylori se deben principalmente a una inapropiada regulación de la respuesta inmune y que la respuesta antiinflamatoria contribuye a la patogénesis del cáncer ${ }^{28,29}$. El supuesto anterior se debe a que dentro del grupo de personas infectadas por $\mathrm{H}$. pylori, las que presentan determinados polimorfismos del gen IL-1B, que codifica la citoquina inflamatoria interleucina-1ß, tienen mayor riesgo de padecer lesiones precancerosas y cáncer gástrico. Esos polimorfismos, en presencia de $\mathrm{H}$. pylori, causan sobre expresión de la proteína IL-1ß, lo que produce una disminución significativa de las secreciones ácidas del estómago $0^{30,31}$, que junto con la hipoclorhidria, crean un ambiente de riesgo de cáncer gástrico ${ }^{32-34}$, que incrementado el daño, permitiendo la acumulación de bacterias y sus toxinas y de mediadores antiinflamatorios.

Se sospecha que el resultado final de la infección está determinado por la extensión y severidad de la inflamación gástrica (gastritis extensiva al cuerpo, hipoclorhidria y atrofia), la cual depende de interacciones complejas entre la edad en que fue adquirida la infección, los mecanismos de respuesta inmune del huésped, la virulencia de la bacteria y la dieta ${ }^{13,25,27,29,34}$.

\section{Historia familiar}

El riesgo de padecer cáncer gástrico es mayor en personas con una historia familiar positiva de cáncer gástrico ${ }^{35-39}$. Esta disposición puede ser el resultado de factores genéticos ambientales. También se ha demostrado que la prevalencia de metaplasia intestinal y de gastritis atrófica e hipoclorhidria es significativamente mayor en familiares de pacientes con cáncer gástrico, infectados por $\mathrm{H}$. pylori, que en controles también infectados ${ }^{40,41}$. B renner et al. (2000) encontraron que la infección por la cepa cagA positiva y una historia familiar de cáncer gástrico, eran factores independientes, y los sujetos que reunían esas dos condiciones tenían 16 veces más riesgo de padecer este tumor ${ }^{38}$.

Como se discutió antes, la herencia de polimorfismos que disminuyen la secreción ácida del estómago (p.e. IL-1B) , en presencia de $\mathrm{H}$. pylori, podría explicar, en parte, el mayor riesgo de padecer cáncer gástrico en las personas infectadas por esa bacteria y con una historia familiar positiva. Estos serían marcadores útiles para identificar individuos con alto riesgo de cáncer gástrico y dirigir los esfuerzos para erradicar la bacteria, o darles seguimiento tendiente a detectar el cáncer en etapa temprana.

\section{Dieta}

La conclusión más consistente sobre el vínculo entre la dieta y el cáncer gástrico, ha sido que las dietas altas en vegetales frescos y frutas reducen el riesgo de cáncer gástrico ${ }^{42-45}$. Esa asociación está especialmente ligada con el consumo de frutas y verduras que contienen antioxidantes. A sí, la menor ingesta de ese tipo de alimentos o los nivel es séricos bajos de beta-carotenos, tocoferoles, y vitamina $C$, están correlacionados con la aparición del cáncer gástrico ${ }^{46-48}$. Sin embargo, las frutas contienen otras sustancias junto con las mencionadas, que pueden ser, por sí mismas, relevantes en la prevención del cáncer. Recientemente, varios estudios han encontrado que el consumo de vegetales del género A llium, especialmente el ajo, disminuyen el riesgo de cáncer gástrico ${ }^{49,50}$. Pero, el consumo de alimentos salados, ahumados, picantes y aquellos que contienen nitrosaminas esta asociado a un mayor riesgo de padecer ese cáncer ${ }^{42,44,45,51-53}$. A Igunos autores sostienen la hipótesis de que los compuestos $\mathrm{N}$-nitrosos, especialmente las nitrosaminas formadas endógena, promueven la carcinogénesis gástrica, mientras que algunos antioxidantes, p.e. el ácido ascórbico, pueden bloquear la preparación de esos compuestos $\mathrm{s}^{43,54}$. 
A demás, los antioxidantes podrían frenar o hacer retroceder las lesiones precancerosas, y evitar que se desarrolle el cáncer gástrico, al reaccionar con los radicales libres 0 agentes oxidantes. Sin embargo, es necesaria mayor investigación, para precisar las funciones de los antioxidantes en el complejo proceso carcinogénico ${ }^{12}$.

\section{Nivel socioeconómico}

Se ha demostrado que la mortalidad por cáncer gástrico está estrechamente relacionada con las condiciones socioeconómicas en los primeros años de la vida ${ }^{55}$. Esta conclusión es consistente con la mayor prevalencia de infección con H. Pylori, en la niñez de poblaciones pobres.

\section{Prevención del cáncer gástrico}

El cáncer gástrico tiene un mal pronóstico, en especial porque la mayoría de los casos se diagnostican en estadíos avanzados, cuando la probabilidad de curarse es muy baja. La prevención primaria de este cáncer debe tener en cuenta la promoción del consumo de frutas y verduras frescas, la reducción del consumo de alimentos picantes, salados e irritantes $y$, en determinados sujetos, la erradicación de H. pylori.

La administración suplementaria de micronutrientes (quimioprevención), para prevenir el cáncer gástrico y otros cánceres, ha sido contradictoria y muy cuestionada ${ }^{56-60}$. A Igunas correlaciones encontradas con la suplementación de antioxidantes, han sido menores que con alimentos, aunque parece que la vitamina C y los beta-carotenos podrían tener algún impacto ${ }^{61-63}$.

En las poblaciones y grupos de alto riesgo deben tomarse medidas audaces, de salud pública, por medio de una "intervención social", promoviendo y facilitando la disponibilidad y consumo de frutas y verduras ricas en antioxidantes, desde la infancia. A demás, hay evidencia de que mejorando el al macenaje y el transporte de los alimentos, se mantendrán mejor su "frescura" y el contenido de antioxidantes, contribuyendo al efecto protector ${ }^{44}$. En poblaciones tropicales con altas tasas de cáncer gástrico, sería fácil promover la producción y consumo de frutas y verduras ricas en vitaminas antioxidantes (mangos, cítricos, papayas, pejibaye, ayotes, piñas, etc), porque tal tipo de alimentos se produce con facilidad en esas áreas. Estas intervenciones tendrían ventajas adicionales y podrían ser beneficiosas, no solo para la prevención de varios cánceres, sino también para disminuir el riesgo de enfermedades cardíacas, diabetes y las relacionadas con el sobrepeso.

También se ha propuesto la erradicación masiva de H. pylori para prevenir el cáncer gástrico ${ }^{64}$. Sin embargo, aunque combinar antibióticos es una buena solución para tratar las patologías gástricas en algunos individuos infectados por H. pylori, ello no representa una solución definitiva. La erradicación masiva de $\mathrm{H}$. pylori para prevenir el cáncer gástric065, es cuestionada por el alto costo, el riesgo de generar cepas resistentes a los antibióticos y por no ofrecer garantías de erradicación definitiva ${ }^{66,67}$. A demás, no ha sido bien valorado el impacto de la erradicación en las lesiones precancerosas, y es difícil predecir cuántos cánceres se evitarían si se erradica $\mathrm{H}$. pylori $25,68,69$. Un buen medio preventivo podría ser la vacunación, que es la práctica médica más efectiva para controlar una infección en la escala global. En todo caso, todavía no se ha aprobado una vacuna, para los humanos, contra esa bacteria ${ }^{70}$. M ientras aparece una vacuna adecuada, se han elaborado criterios que establecen, según las diferentes poblaciones, cuáles son los sujetos que deben recibir tratamiento ${ }^{61,71-75}$. A demás, los recientes aportes de El-O mar y colaboradores ( 2000 y 2001), en relación con los polimorfismos genéticos del hospedero con mayor riesgo de desarrollar cáncer, podrían orientar aún más sobre los sujetos infectados que deberían recibir tratamiento contra la bacteria ${ }^{31,41}$.

Es importante considerar que para prevenir el cáncer gástrico, posiblemente no será suficiente eliminar un sólo factor de riesgo, porque el cáncer gástrico ocurre a través de un proceso multifactorial y con diferentes estadios. Para elaborar e implementar un programa de prevención, será necesaria la participación de un grupo multidisciplinario de científicos y clínicos que enfrenten el problema de una forma integral.

\section{Detección temprana}

Los programas de detección se basan en el supuesto de que el cáncer temprano (aquel que está circunscrito a la mucosa y submucosa), si no se trata, puede progresar a cáncer avanzado, y luego a la muerte del paciente.

En Japón, donde el cáncer gástrico es un problema importante de salud pública, se han implantado programas masivos de detección temprana, por medio de fotofluorografía. Los pacientes sospechosos de presentar una lesión cancerosa detectados por Rayos $X$, se someten a una gastroscopía y biopsia. Los casos con cáncer se someten a una cirugía. Esos programas han tenido un éxito reconocido ahí76-78, pero, se ha cuestionado la baja especificidad del método y su costo-efectividad. Es un proceso caro porque requiere de un grupo de médicos y técnicos especializados y de equipos de alta tecnología $a^{79.81}$. A demás, ese sistema debe ser adaptado a las circunstancias socioeconómicas y culturales de los países o grupos donde se trata de instalar ${ }^{82}$. Es importante hacer énfasis en la necesidad de que los programas de detección sean económicos, simples y efectivo ${ }^{83}$, especialmente para países pobres, con altas tasas de cáncer gástrico. Algunos han cuestionado si todos los cánceres tempranos finalizan en cáncer invasor ${ }^{84}$, por lo cual es importante determinar el impacto de los programas de detección en las tasas de mortalidad por este tipo de cáncer.

Hace poco tiempo, se introdujo en Japón la prueba de pepsinógenos séricos, se basa en que los niveles de pepsinógenos I y II (PG I y PG II) en suero reflejan el estado 
funcional y morfológico de la mucosa gástrica ${ }^{85.86}$. Varios estudios muestran que valores bajos de la razón PGI/PG II significan al to riesgo de gastritis crónica atrófica y cáncer gástrico, y la determinación de estos niveles puede ser usada

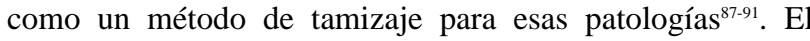
sistema tiene muchas ventajas, porque es sencillo y barato, aunque antes de usarlo masivamente, se deben determinar, en la población donde se va a emplear, los puntos de corte (cut off ) de los niveles séricos de PGs I y II, que discriminan las diferentes lesiones precancerosas y cáncer. Después sería necesario evaluar el impacto del programa que usa estos marcadores, en las tasas de mortalidad por cáncer gástrico. Se ha demostrado que la sensibilidad de la detección masiva se incrementa cuando se combinan la determinación de los niveles de PGs séricos con la serie gastroduodenal de doble contraste ${ }^{80}$. M uchas personas participarían con mayor interés, en los programas de detección, si los resultados de una prueba de sangre indican que son sujetos de alto riesgo de cáncer gástrico ${ }^{92}$. La identificación de grupos de alto riesgo, por la historia familiar marcadores biológicos, podría disminuir el costo y mejorar la efectividad del método de detección de cáncer gástrico.

En Táchira, Venezuela, se encontró que la detección temprana de cáncer gástrico, de acuerdo con el modelo japonés tradicional, tuvo un efecto protector y disminuyó la mortalidad por cáncer gástrico, en el grupo tamizado ${ }^{93-94}$.

Costa Rica es uno de los países con mayores tasas de inicidencia y mortalidad por cáncer gástrico. En el período 1995-2000, se llevó a cabo un programa piloto de detección temprana, en una muestra de alto riesgo (Hospital Max Peralta), con la colaboración de Japón ${ }^{95}$. A ctualmente, nuestro equipo está llevando a cabo un estudio epidemiológico basado en la información generada por ese programa. También se está evaluando el costo-efectividad del mismo, con el fin de elaborar alternativas para mejorarlo e investigamos marcadores de lesiones gástricas precancerosas y cáncer (pepsinógenos séricos I y II) y factores genéticos (polimorfismos de interleucinas), así como las diferentes cepas de $H$. pylori en la población costarricense (Hospital Max Peralta, Hospital Calderón Guardia). Se trata de encontrar marcadores biológicos para identificar a los sujetos y grupos con mayor riesgo de cáncer gástrico en el país, y determinar el método más adecuado para darles seguimiento o para tratarlos. El objetivo final es encontrar un sistema de detección adecuado al contexto socioeconómico y cultural de Costa Rica, con el fin de disminuir significativamente la mortalidad por ese tumor.

\section{Agradecimientos}

A gradezco a los Drs. J. Jaramillo, C. A. Páez, C. Une, L. Lang y $\mathrm{F}$. García, la revisión del artículo, y el apoyo de la Vicerrectoría de Investigación de la Universidad de Costa Rica, al Programa E pidemiología de Cáncer.

\section{Abstract}

Gastric cancer is one of the tumors with higher worldwide mortality. A lthough the incidence rate has been declining, it is still a major public health problem still. Epidemiological information and knowledge about this cancer is abundant. This is an up to date review about commonly accepted risk factors: Helicobacter pylori infection, diet, genetic predisposition and socioeconomic status. We also stress the new questions and challenges that recent discoveries about this pathology entail. The ways to tackle this cancer are discussed using $X$ rays and biological markers such as serum pepsinogens in high risk groups or populations.

\section{Referencias}

1. Coleman M.P, Esteve J, Damiecki P, Arslan A, Renard H. Trends in Cancer Incidence and M ortalily. Lyon, 1993: International A gency for Research on Cancer, (IARC/WHO).

2. Parkin DM , Pisani P, Ferlay J. Estimates of the worldwide incidence of 25 major cancers in 1990. Int J Cancer ,1999; 80:827-841.

3. Pisani P, Parkin DM, B ray F, Ferlay J. Estimates of the worldwide mortality from 25 cancers in 1990. Int J Cancer, 1999; 83:18-29.

4. Douglas H. Potentially Curable Cancer of the Stomach. Cancer, 1982; 50: 2582-2589.

5. Webb A, Cunningham D. Curing gastric cancer - home the scal pel with magic?. Br J Cancer 1996; 73:418-419.

6. Hisamichi S, Sugawara N, Fukao A. Effectiveness of gastric mass screening in Japan. Cancer Detect. Prev, 1988, 11:323-329.

7. Fukao A, Tsubono Y, Tsuji I. The evaluation of screning of gastric cancer in Miyagi Prefecture, Japan: a population-based case-control study. Int J Cancer, 1995a; 60:45-48.

8. Standtlander Ch T, Waterbord JW. Molecular Epidemiology, pathogenesis and prevention of gastric cancer. Review. Carcinogenesis, 1999; 20:2195-2207.

9. Yokota T, Takahashi N, Teshima S, Yamada $Y$, Saito T,Kakizaki K. Early Gastric Cancer in the Young: Clinicopathological Study. A ust N Z Surg, 1999; 69:433-446

10. Park J, Yang H, Yo K in W, Caldas $\mathrm{C}$. Report on the first meeting of the international collaborative group on hereditary gastric cancer. J Natl Cancer Inst, 2000; 92(21):1781-1782.

11. Correa P, Haenzsel W, Cuello C, Zavala D, Fontham E, Zamara G, Tannenbaum S, Collazos T, Ruiz B. Gastric precancerous proccess in a high risk population: cohort follow-up. Cancer Res, 1990; 50:47374740 .

12. Correa P. Human Gastric carcinogenesis : a multistep and multifactorial proces Cancer Res, 1992; 52:6735-6740.

13. Forman D. Helicobacter pylori and Gastric Cancer. Scand J Gastroenterol, 1996; 215:48-51.

14. Blaser M. Helicobacter pylori and gastric diseases. Science, medicine and the future. Clinical Review. BMJ, 1998; 316:1507-1510.

15. Kandel G. Helicoabacter and disease: still more questions than answers. Can J Surg, 2000; 43:339-346. 
16. Imrie C, Rowland M, Bourke B, Drumm B. Is Helicobacter pylori infection in Childhood a Risk Factor for Gastric Cancer?. Pediatrics, 2001; 107:373-380.

17. Logan RP, Walker MM. Epidemiology and diagnosis of Helicobacter pylori infection. Clinical review. B M J, 2001; 323:920-922.

18. Correa P. H. pylori and Gastric Carcinogenesis. Am J Surg Pathol, 1995; 19(Supp1):S37-S43.

19. Dunn $B$, Cohen $H$, Blaser M. Helicobacter pylori. Clin M icobiol Rev, 1997; 10:720-741.

20. Forman D. Helicobacter pylori, infection and cancer. Br M ed Bull, 1998; 54:71-78.

21. Blaser M.J, Pérez-Pérez G, Kleanthous H, Cover T. Chyou PR, Stemmerman $\mathrm{G}$. and Nomura $\mathrm{A}$. Infection with Helicobacter pylori strains possessing cagA is assciated whith an increased risk of developing adenocarcinoma of the stomach. Cancer Res, 1995; 55:2111-2115.

22. Rokkas T, Liatsos C, Petridou E, Papatheodoru S, Karameris A, Ladas $\mathrm{S}$, Raptis S. Relationship of $\mathrm{H}$. pylori CagA (+) status to gastric juice vitamin C levels. Eur J Clin Invest, 1999; 29:56-61.

23. M aeda $S$, Ogura $K, Y$ oshida $H, K$ anai $F$, Ikenoue $T, K$ ato $N$, Shiratori Y, O mata M. M ajor virulence factors, VacA and CagA, are commonly positive in Helicobacter pylori isolates in Japan. Gut, 1998; 42:338343.

24. Webb PM, Grabtree JE, Forman D and The Eurogast Study Group. Gastric Cancer, Cytotoxin-A ssociated Gene A-Positive Helicobacter pylori, and Serum Pepsinogens: an International Study. Gastroenterology, 1999; 116:269-276.

25. Calam J, Baron J. Pathophysiology of duodenal and gastric ulcer and gastric cancer. ABC of the upper gastrointestinal tract. BMJ, 2001; 2323:980-982.

26. Evans DJ, Evans DG. Helicobacter pylori CagA : A nalysis of Sequence Diversity in Relation to Phosphorylation Motifs and Implications for the Role of CagA as a Virulence factor. Helicobacter, 2001; 6(3):186198.

27. U emura N, Okamoto $S$, Yamamoto $S$, Matsumura N, Yamaguchi $S$, Yamakido N, Taniyama K. Helicobacter pylori Infection and the Development of Gastric cancer. N ew Engl J M ed, 2001; 345:784-789.

28. Ernst $P$. Review article: the role of inflammation in the pathogenesis of gastric cancer. Aliment Pharmacol Ther, 1999; 13(Suppl.1):13-18.

29. Ernst P, Gold B. The disease spectrum of Helicobacter pylori: The inmunopathogenesis of gastroduodenal ulcer and gastric cancer. Ann Rev Microbiol, 2000; 54:615-640.

30. El-Omar E.M. Carrington M., Chow WH, M cColl K, B ream J, young H, Herrera J, Lissowska J, Yuan Ch, Rotman N, Lanyon G, M artin M, Fraumeni J, Rabkin L. Interleukin-1 polymorphism associated with increased risk of gastric cancer. Nature, 2000a; 404:398-402.

31. El-Omar E.M. The importance of interleukin 1 B in Helicobacter pylori associated disease. Leading article. Gut, 2001; 48:743-747.

32. M oore C. Helicobacter pylori -cancer link explained by host genes. The Lancet, 2000; 355:1079.

33. Balkwiil F, M antovani A . Inflammation and Cancer: back to Virchow? Review Lancet, 2001; 357:539-546.

34. Kiupers EJ. Review article: exploring the link between Helicobacacter pylori and gastric cancer. Aliment Pharmacol Ther, 1999; 13(Suppl1):3-11.
35. Jackson C, Brownlee R, Shuman B, Micheloni F, Ghironzi G. Observations on Gastric Cancer in San Marino. Familial Factors. Cancer, 1980; 45:599-602.

36. La Vecchia C, Negri E, Franceschi S, Gentile A. Family history and the risk of stomach and colorectal cancer. Cancer ,1992; 70:50-55.

37. Palli D, Galli M, Caporaso E, Cipriani E, Decarli A, Saieva C, Fraumeni $\mathrm{J}$, B uiati E. Family History and Risk of Stomach Cancer in Italy. Cancer Epidemiology, Biomarkers and Prevention, 1994; 3:15-18.

38. Inoue M, Tajima K, K obayashi S, Suzuki T, M atsuura A, Nakamura T, Inuzuka K, Shirai M, Nakamura S, Tominaka S. Prospective factor against progression from atrophic gastritis to gastric cancer- data from a cohort study in Japan. Int J Cancer, 1996; 66:309-314.

39. B renner $\mathrm{H}$, A rndt $\mathrm{V}$, Sturmer $\mathrm{T}$, Stegnnaier $\mathrm{Ch}$, Zeigler $\mathrm{H}$, Dhom $\mathrm{G}$. Individual and Joint Contribution of Family History and Helicobacter pylori Infection to the Risk of Gastric Carcinoma. Cancer, 2000; 88:274-279.

40. M eining A G, B ayerdörffer $E$, Stolte M . Helicobacter pylori gastritis of the gastric cancer phenotype in relatives of gastric carcinoma patients. Eur J Gastroenterol Hepathol, 1999; 11:717-720.

41. El-Omar E, Oien K, Murray L, El-Nujumi A, Wirz A, Gillen D, Willians C, Fullarton G, MCColl K. Increased Prevalence of Precancerous Changes in Relatives of Gastric Cancer Patients: Critical Role of H. pylori. Gastroenterology, 2000b; 118:22-30.

42. Buiatti $E$, Palli $D$, Decarli $D, A$ madori $D$, A vellini $C, B$ ianchi $S, B$ iserni $R$, Cipriani $F, C$ occo $P$, Giacosa $A, M$ arubini $E$, Puntoni R, Vindigni $C$, Fraumeni J, B lot W .A case-control Study of Gastric Cancer and Diet in Italy. Int J Cancer, 1989; 44:611-616.

43. Correa P. The role of Antioxidants in gastric Carcinogenesis. Critical Rev Food Sci Nutr, 1995; 35(1\& 2):59-64.

44. Gastric cancer Panel: B erglund G, Hill M, Caygill C, Farinati F, Giacosa A, De Koster E, Reed P, Sobrinho-Somoes M, Strockbrougger R.Consensus Statement on diet and gastric cancer. Eur J Cancer Prevention, 1997; 6:404-407.

45. Hensrud DD, Heimburger D.C. Nutritional, Physiologic, and Pathophysiologic Considerations of the Gastrointestinal Tract. Diet, Nutrients, and Gastrointestinal Cancer. Gastroenterol Clin, 1998; 27(2):326-351.

46. Tsugane S, Kabuto M, Imai H, Gey F, Tei Y, Hanaoka T, Sugano K, Watanabe $\mathrm{S}$. Helicobacter pylori, dietary factors, and atrophic gastritis in five Japanese populations with different gastric cancer mortality. Cancer Causes and Control, 1993; 4:297-305.

47. Zhang L, B lot W, Y ou W, Chang Y, Liu X, K neller W, Zhao L, Liu W, $L i J$, J in $M, X u G$, Fraumeni $F J r$, Yang $S$. Serum micronutrients in relation to precancerous gastric lesions. Int J Cancer, 1994; 56: 650654.

48. A merican Institute for Cancer Research. Stomach. En: Food, Nutrition and the Prevention of Cancer: a global prespective. USA : World Cancer Research Fund, 1997: 148-175.

49. Sivam GP, Lampe JW, U Iness B, Swanzy SR, Potter JD. Helicobacter pylori -in vitro susceptibility to Garlic (Allium sativum) Extract. Nutr Cancer, 1997; 27(2):118-121.

50. Fleischauer AT, Poole Ch, A rab L. Garlic consumption and cancer prevention: meta-analyses of colorectal and stomach cancer. A mer J Clin Nutr, 2000; 72:1047-52.

51. Correa P, Fontham E, Pickle LW, Chen V, Lin Y, Haenzel W. Dietary Determinants of Gastric Cancer in South L ouisiana Inhabitants. J NCl, $1985 ; 75: 645-654$. 
52. Hu J, Zhang S, Jia E, Wang Q, Liu S, Liu Y, W uY, Chen Y. Diet and Cancer of the Stomach: a case-control Study in China. Int J Cancer, 1988; 41:331-335.

53. Hill M J. Salt and gastric cancer. Editorial. Eur J Cancer Prevention, 1998; $7:$ 173-175

54. Sierra R, Chinnock A, Oshima H, Pignatelli B, M alaveille $C h, \mathrm{Gamboa}$ G, Teuchmann S, Muñoz N, Bartsch H. In Vivo Nitrosoproline Formation and Other Risk Factors in Costa Rican Children from Highand Low-Risk A reas for Gastric Cancer. Cancer Epidemiol., Biomarkers\& Prev., 1992; 1:449-454

55. Forman D, Goodman KJ. The epidemiology of stomach cancer correlating the past with the present. Socioeconomic influences in early life mortality in adult life (editorial coment). BM J, 2000; 320:1682 1683.

56. M iller $A, B$ errino $F$, Hill $M$, Pietinen $P$, Riboli $E$, and Waherendorf $M$. Diet in the A etiology of Cancer: Review. Eur J Cancer, 1994; 30:207220

57. O 'Toole P, L ombard M . Vitamin C and gastric cancer: supplements for some or fruits for all?. Leading article. Gut, 1996; 39:345-347.

58. Paolini M, Cantelli-Forti G, Perocco P, Pedulli G, A bdel-Rahman Z, Legator M. Co-carcinogenic effect of ß-carotene. Nature, 1999; 398:760-761.

59. Deblinger L. Nutrition and Cancer Prevention. Patient Care, 2001; $35: 21-30$

60. Y ou W, Zhang L, G ail M, Chang Y. G astric displasia and gastric cancer: Helicobacter pylori serum vit. $C$ and other risk factors. J Natl Cancer Inst, 2000; 92:1607-1612.

61. Buiatti $E, M$ uñoz N. Chemoprevention of stomach cancer. In: Hakama M, Beral V, Buiatti E, Faire J, Parkin DM, eds. Chemoprevention in Cancer Control. IARC Scientific Publications No. 136. Lyon: International A gency for Research on Cancer, 1996:35-36.

62. Correa P, Fontham ET, Bravo J, B ravo L, R uiz B, Zarama G, Real pe J L, $M$ alcom D, Li D, Jhonson W D, M era R. Chemoprevention of Gastric Dysplasia: Randomiced Trial of Antioxidant Supplements and AntiHelicobacter pylori Therapy. J Natl Cancer Inst, 2000; 92: 1881-1888.

63. Zullo A, Rinaldi V, Hassan C, Diana F, Winn S, Castagna G, A ttili A A scorbic acid and intestinal metaplasia in the stomach: a prospective, randomized study. Aliment Pharmacol Ther, 2000; 14:1303-1309.

64. Parsonnet J, Harris R, Hack H, O wens D. M odelling cost-effectiveness of $\mathrm{H}$. pylori screening to prevent gastric cancer: a mandate for clinical trials. The Lancet, 1996; 348:150-154.

65. Covacci A, Telford J, Del Giudice G, Parsonnet J, Rappuoli R Helicobacter pylori virulence and genetic geography. Science, 1999; 284:1328-1333.

66. Blaser MJ. Helicobacter pylori and gastric diseases. Clinical review. Science, medicine, and the future. BMJ, 1998; 316:1507-1510.

67. Blaser M.J. In a World of Black and White, Helicobacter pylori Is Gray? . A nn Intern M ed , 1999; 130(8):695-697.

68. Danesh J, Pounder R. Eradication of $\mathrm{H}$. pylori and non-ulcer dyspepsia. The Lancet, 2000; 355: 766-767.

69. Fox JG, Wang TC. Helicobacter pylori- Not a good bug after all. Editorial. N Engl J M ed, 2001; 345:829-831

70. Walt B, Kerr G. Can eradicating H. pylori prevent gastric cancer? The L ancet, 1998; 351:887.

60 AM C, abril-junio 2002, vol 44 (2)
71. Goodwin CS, M endakI M M, Northfield TC. H. pylori infection. The Lancet, 1997: 349:265-269.

72. Coelho $L$, León-Barúa $R$, Q uigley $M$, and representatives of the $L$ atin A merican National Gastroenterology Latin-A merican Consensus Conference on Helicobacter pylori Infection. Special Article. AJG, 2000; 95:2688-2691.

73. Peterson WL, Fendrick AM, Cave DR, Perua DA, GarambediannRuffalo M, Laine L. Helicobacter pylori- Related Disease. A rch Intern Med, 2000; 160(9):1285-1291.

74. Bazzoli F. K ey points from the revissed $M$ aastrich Consensus Report: the impact on general practice. Eur J Gastroenterol Hepatol, 2001; 13 (suppl 2):S3-S7.

75. Harris A, M isiewicz JJ. M anagement of Helicobacter pylori infection. ABC of the upper gastrointestinal tract. Clinical review. BMJ, 2001; 323:1047-1050

76. Oshima A, Hanai A, Fujimoto I. Evaluation of a Mass Screening Program for Stomach Cancer. N atl Cancer Inst M onogr, 1978; 53:181186

77. Hisamichi S. Screening for G astric Cancer. W orld J Surg, 1989; 13:31-37.

78. Fukao A, Tsubono Y,T suji I, Hisamichi S, Sugahara N, Takano A. The evaluation of screening for gastric cancer in M iyagi Prefecture, Japan : a population-based case control study. Int J Cancer, 1995b; 60:45-48.

79. Kodoi A, Yoshihara M, Sumi K, Hamura K, Kajiyama G. Serum pepsinogen in screening for gastric cancer. J Gastroenterol, 1995; 30:452-460.

80. Yoshihara M, Sumii K, Haruma K, Kiyohira K, Hattori N, Tanaka S, Kajiya $G$, Shigenobu T. The U sefulness of Gastric Mass Screening Using Serum Pepsinogen Level Compared with Photofluorography. HiroshimaJ Med Sci, 1997; 46(2):81-86.

81. Kitahara F, Kobayashi K, Sato T, Kojima $Y$, A raki T, Fujino M Accuracy of Screening for gastric cancer using serum pepsinogen concentrations, Gut, 1999; 44:693-697.

82. Sano T. Managing gastric cancer in Britain: a Japanese experience. Personal view. BM J, 1997; 314:382.

83. Yoshida S, Saito D. Gastric premalignancy and cancer Screening in High-Risk Patients. World Congres of Gastroenterology, L os A ngeles, 1994. A m J Gastroenterol, 1996; 91:839-848.

84. Everett SM, Axon AT. Early gastric cancer: disease or pseudo-disease? Viewpoint. The L ancet, 1998; 351:1360-1352.

85. Samloff IM. Varis K, Ihamaki T, SiuralaM, and Rotter J. Relationship among serum pepsinogen I, serum pepsinogen II and gastric mucosal histology: a study in relatives of patients with pernicious anemia. Gastroenterology, 1982; 83:204-209 .

86. Miki $\mathrm{K}$, Ichinose $\mathrm{M}, \mathrm{K}$ awamura $\mathrm{N}$, M atsushima $\mathrm{M}$, A hman $\mathrm{H}$, K imura M, Sano J, Tashiro T , Kakei N, Oka H, Furihata C and Takahashi. The Significance of Low Serum Pepsinogen Levels to Detect Stomach Cancer Associated with Extensive Chronic Gastritis in Japanese Subjets. Jpn J Cancer Res, 1989; 80:111-114.

87. M iki K, Ichinose $M$, Ishikawa $K$, Yahagi $N$,, M atsushima $M, K$ akei $N$, Tsukada S, Kido M, Ishihama S, Shimizu Y Susuki T, Kurokawa K. Clinical A plication of Serum Pepsinogen I and II Levels for Mass Screening to Detect Gastric Cancer. J pn J .Cancer Res, 1993; 84: 10861090.

88. Kodoi A, Yoshihara M, Sumii K, Haruma K, Kajiyama G. Serum pepsinogen in screening for gastric cancer. J Gastroenretol, 1995; 30:452-460. 
89. A oki K, M isumi J, Kimura T, Zhao W, and XieT. Evaluation of Cuttoff Levels for Screening of gastric Cancer Using Serum Pepsinogens and Distributions of Levels of Serum Pepsinogen I, II and of PGI/PGII Ratios in a Gastric Cancer Case-Control Study. J Epidemiol, 1997; 7:143-151.

90. Yoshihara M, Sumuii K, Haruma K, Haruma K, K iyohira K, Hattori N, K itadai $Y, K$ omoto $K$. Tanaka S, Kajiyama N. Correlation of Ratio of Serum Pepsinogen I and II With Prevalence of Gastric Cancer and A denoma in Japanese Subjets. Am J Gastroenterol, 1998; 93:10901096

91. Kitahara $F$, Kobayashi $K$, Sato $T, K$ ojima $Y$, A raki $T$, Fujino M. Accuracy of screening for gastric cancer using serum pepsinogen concentrations. Gut, 1999; 44:693-697.

92. Kurosawa M, Kikuchi S, A riuse T, Fukao A. Effectiveness and feasibility of a strategy for increasing participation in the Japanese Stomach Cancer Examination programs by incorporating serum pepsinogen tests. Nippon K oshu eisei Zasshi, 1998; 45:352-360

93. Pisani $\mathrm{P}$, Oliver WE, Parkin DM, Alvarez N, Vivas J, Case-control study of gastric cancer screening in Venezuela. $\mathrm{Br}$ J Cancer, 1994; 69:45-48.

94. Parkin DM , Pisani P. Gastic Cancer. In: K ramer B, Gohagan J, Prorok $P$ eds. Cancer Screening. Theory and Practice. New York: Marcel Dekker, Inc, 1999: 515-529.

95. Sasagawa T, Solano H, Mena F. Gastric Cancer in Costa Rica. Gastrointestinal Endoscopy, 1999; 50:4. 